\title{
Atividades farmacológicas dos extratos de Zanthoxylum chiloperone (Rutaceae)
}

\author{
Milcíades A. Villalba, Maria I. Carmo, Magda N. Leite, Orlando V. Sousa* \\ Departamento Farmacêutico, Faculdade de Farmácia e Bioquímica, Universidade Federal de Juiz de Fora, \\ Campus Universitário, 36036-330, Juiz de Fora, MG, Brasil
}

\begin{abstract}
RESUMO: Neste trabalho foram avaliadas as atividades antinociceptiva e antiinflamatória dos extratos das folhas de Zanthoxylum chiloperone. Os ensaios foram realizados pelos testes de contorções abdominais induzidas por ácido acético, tempo da lambida da pata induzida por formalina, edema de pata e pleurisia induzidas por carragenina, usando os extratos hexânico, em acetato de etila e etanólico administrados nas doses de 100 e $200 \mathrm{mg} / \mathrm{kg}$. As doses testadas reduziram, significativamente, as contorções abdominais, o tempo de lambida da pata, o edema de pata, o volume do exsudato e a migração leucocitária. Estes resultados sugerem que os extratos de Z. chiloperone possuem constituintes com atividades antinociceptiva e antiinflamatória.
\end{abstract}

Unitermos: Zanthoxylum chiloperone, atividade antinociceptiva, atividade antiinflamatória.

\begin{abstract}
Pharmacological activities of Zanthoxylum chiloperone (Rutaceae) extracts”. In this work the antinociceptive and anti-inflammatory activities of the extracts of leaves from Zanthoxylum chiloperone were evaluated. The tests were carried out by acetic acid writhing, formalin-induced paw licking, carrageenan-induced rat paw edema and pleurisy methods, using hexane, ethyl acetate and ethanol extracts administered in the doses of 100 and $200 \mathrm{mg} / \mathrm{kg}$. The doses inhibited, significantly, the contortions, the paw lick, the paw edema, the volume of the exsudate and the leukocytes migration. These results suggest that the extracts from Z. chiloperone possess constituents with antinociceptive and anti-inflammatory activities.
\end{abstract}

Keywords: Zanthoxylum chiloperone, antinociceptive activity, anti-inflammatory activity.

\section{INTRODUÇÃO}

Espécies do gênero Zanthoxylum são utilizadas na medicina popular no tratamento de doenças cardiovasculares, tuberculose, malária, para aliviar dor de dente, contra mordida de cobra e como analgésico (Weenen et al., 1990; Arruda et al., 1992; Gessler et al., 1994; Vigneron et al., 2005). Investigações fitoquímicas identificaram alcalóides, flavonóides, cumarinas, lignanas e terpenos no gênero (Talapatra et al., 1973; Stermitz; Sharifi, 1977; Diehl et al., 2000; Oliveira et al., 2002; Facundo et al., 2005; Tane et al., 2005). Propriedades antinociceptiva, antidiarréica, antimalárica, antioxidante, mutagênica, antimicrobiano, giardicida, entre outras, têm sido demonstradas em espécies de Zanthoxylum (Rahman et al., 2002; Tatsadjieu et al., 2003; Chung et al., 2006; Jullian et al., 2006; Amaral et al. 2006).

Zanthoxylum chiloperone Mart. Engl. é uma Rutaceae encontrada no Sudeste e Sul do Brasil e na América do Sul (Albuquerque; Rodrigues, 2000; Silva; Soares, 2003). Tribos indígenas do Paraguai usa a espécie como antimalárico, enemagogo, anti-reumático, antimicrobiano e para dor de cabeça (Milliken 1997; Guy et al., 2001). Estudos fitoquímicos de Z. chiloperone identificaram alcalóides e óleos essenciais (Soriano-
Agatón et al., 2005; Thouvenel et al., 2003; Ferreira et al., 2002). Atividades leishmanicida e antifúngica (Thouvel et al., 2003; Ferreira et al., 2002), assim como tripanomicida (Ferreira et al., 2007), foram atribuídas a substâncias isoladas desta espécie.

Devidos suas indicações para dor e como antireumático, assim como os usos medicinais de espécies do gênero, o presente trabalho teve como objetivo estudar as atividades antinociceptiva e antiinflamatória dos extratos das folhas de $Z$. chiloperone através de modelos de nocicepção e de inflamação.

\section{MATERIAL E MÉTODOS}

\section{Coleta do material vegetal}

Zanthoxylum chiloperone foi coletada em janeiro de 2004 na cidade de Concepción (Paraguai). Uma exsicata, identificada por Dra. Fátima Regina Gonçalves Salimena, foi depositada no Herbário Leopoldo Krieger do Departamento de Botânica da Universidade Federal de Juiz de Fora (UFJF) sob $n^{\circ}$ CESJ 41.683, Juiz de Fora, Minas Gerais, Brasil.

\section{Preparo do extrato}


As folhas foram submetidas à secagem a 50 ${ }^{\circ} \mathrm{C}$, sob ventilação forçada, até perda de 90 a $95 \%$ de sua umidade. $\mathrm{O}$ material botânico foi triturado em moinho mecânico e pulverizado em tamise de malha 80. A extração do material pulverizado (100 g para cada solvente) foi feita em aparelho de Soxhlet, usando hexano, acetato de etila e álcool $70 \%$ por 24 horas, separadamente, produzindo os extratos hexânico (EH), em acetato de etila (EA) e etanólico (EE). Os solventes foram removidos em rotavapor para obtenção dos resíduos secos. Todos os extratos foram solubilizados em dimetilsulfóxido (DMSO) $1 \%(\mathrm{v} / \mathrm{v})$ em salina para avaliar as atividades farmacológicas.

\section{Animais}

Foram utilizados camundongos Swiss (25-30 g) machos eratos Wistar (180-240 g) machos provenientes do Centro de Biologia da Reprodução da UFJF. Os animais foram mantidos em gaiolas plásticas com ração e água ad libitum em temperatura ambiente. Doze horas antes da realização das experiências, os animais foram privados de ração. Os protocolos utilizados foram aprovados pelo Comitê de Ética na Experimentação Animal (CEEA) desta instituição (Processo Nº. 020/2005).

\section{Avaliação da atividade antinociceptiva}

\section{Teste de contorções abdominais}

Os extratos de $Z$. chiloperone foram administrados (100 e $200 \mathrm{mg} / \mathrm{kg}$ ), por via oral (v.o.), 1 hora antes da aplicação do ácido acético $0,6 \%(\mathrm{n}=8)$ (Koster et al., 1959). Uma hora após tratamento, $10 \mathrm{~mL} / \mathrm{kg}$ de ácido acético $0,6 \%$ foram administrados intraperitonealmente em cada camundongo e $\mathrm{o} \mathrm{n}^{\circ}$ de contorções abdominais contado entre 10 e 30 minutos após este procedimento. O grupo controle recebeu $10 \mathrm{~mL} / \mathrm{kg}$ de DMSO 1\% v/v em salina (v.o.). A indometacina (10 mg/kg), controle positivo do teste, foi administrada por via oral.

\section{Teste da formalina}

Injeção de $20 \mu \mathrm{L}$ de formalina 2,5\% (em salina estéril) foi aplicada no espaço subplantar da pata direita de camundongos $(n=8)$ e a duração da lambida foi determinada de 0 a 5 minutos ( $1^{\text {a }}$ fase) e 15 a 30 minutos ( $2^{\mathrm{a}}$ fase) após aplicação da formalina (Hunskaar; Hole, 1987). Os extratos de $Z$. chiloperone foram administrados nas doses de 100 e $200 \mathrm{mg} / \mathrm{kg}$ (v.o.) 1 hora antes da injeção da formalina. Os animais controle receberam 10 $\mathrm{mL} / \mathrm{kg}$ de DMSO $1 \%$ em salina (v.o.). Morfina (1 mg/kg, subcutânea) foi usada como controle positivo teste.

\section{Avaliação da atividade antiinflamatória Teste do edema de pata induzido por carragenina}

O edema de pata foi induzido pela injeção de $0,1 \mathrm{~mL}$ de carragenina $(2 \% \mathrm{p} / \mathrm{v})$ em salina estéril e administrada na região subplantar da pata direita de rato Wistar machos $(n=6)$. Uma hora antes da injeção de carragenina, os extratos Z. chiloperone, solubilizados em DMSO $1 \% \mathrm{v} / \mathrm{v}$ em salina, foram administrados (v.o.) nas doses de 100 e $200 \mathrm{mg} / \mathrm{kg}$ (Winter et al., 1962). O grupo controle recebeu $10 \mathrm{~mL} / \mathrm{kg}$ de DMSO $1 \%$ em salina (v.o.), correspondendo a cerca de $85 \mathrm{mg} / \mathrm{kg}$ de $\mathrm{NaCl} 0,85 \%$. Na pata esquerda, usada como controle, foi injetada $0,1 \mathrm{~mL}$ de salina estéril. Após quatro horas de injeção de carragenina, a medida do edema foi feita pela diferença entre o volume deslocado da pata direita e o da pata esquerda. Indometacina (10 mg/kg, v.o.) foi usada como controle positivo do teste.

\section{Teste da pleurisia induzida por carragenina}

A pleurisia foi induzida em ratos Wistar pela injeção intrapleural de $0,5 \mathrm{~mL}$, entre a terceira e a quinta costela do lado direito do mediastino, de uma suspensão de carragenina 2\% em solução salina estéril (Vinegar et al., 1973). Grupos de seis ratos foram tratados com extratos de Z . chiloperone (100 e $200 \mathrm{mg} / \mathrm{kg}$, v.o.), indometacina (10 mg/kg, v.o.) ou $10 \mathrm{~mL} / \mathrm{kg}$ de DMSO $1 \% \mathrm{v} / \mathrm{v}$ em salina (v.o.) uma hora antes da aplicação do agente inflamatório. Quatro horas após a indução da inflamação, os animais foram anestesiados com cloridrato de cetamina $(75 \mathrm{mg} /$ $\mathrm{kg})$ e cloridrato de xilazina $(10 \mathrm{mg} / \mathrm{kg})$ e sacrificados. Uma incisão foi feita entre a terceira e quinta costela em cada lado do mediastino. O exsudato pleural foi coletado, transferido a um tubo cônico de centrífuga e o volume foi determinado. Uma alíquota de $50 \mu \mathrm{L}$ do exsudato foi usada para determinar a contagem total de leucócitos em câmara de Neubauer. Realizou-se também a contagem de polimorfonucleares e mononucleares em esfregaços preparados em lâminas e corados com Leishmann.

\section{Análise estatística}

Os resultados foram demonstrados através da média \pm erro padrão. Análise de variância (ANOVA) seguida do teste de Student Newman-Keuls foi utilizada para medir o grau de significância $(\mathrm{p}<0,05)$.

\section{RESULTADOS}

O teste de contorções abdominais demonstrou que os extratos das folhas de $Z$. chiloperone inibiram as contorções nas doses administradas. Os extratos causaram inibição de 79,67\% (EH), 75,41\% (EA) e 78,72\% (EE) na dose de $200 \mathrm{mg} / \mathrm{kg}$. A indometacina inibiu 76,36\% as contorções, mostrando sua eficácia como analgésico (Figura 1).

A injeção de 2,5\% de formalina promoveu uma resposta característica bifásica (Tabela 1). A duração 




Figura 1. Efeitos dos extratos de Z. chiloperone sobre o número de contorções abdominais induzidos por ácido acético (0,6\%) em camundongos $(\mathrm{n}=8)$. Indometacina (Indomet), $10 \mathrm{mg} / \mathrm{kg}$, foi o controle positivo teste. ${ }^{* * *} \mathrm{P}<0,001$. Significativos após análise de variância (ANOVA) seguido do teste de Student Newman-Keuls quando comparados ao controle.

Tabela 1. Efeitos dos extratos de Z. chiloperone no modelo do tempo de lambida da pata induzida por formalina $2,5 \%(\mathrm{n}=8)$.

\begin{tabular}{|c|c|c|c|c|c|}
\hline \multirow{2}{*}{$\begin{array}{l}\text { Grupos } \\
\text { experimentais }\end{array}$} & \multirow{2}{*}{$\begin{array}{c}\text { Doses } \\
(\mathrm{mg} / \mathrm{kg})\end{array}$} & \multicolumn{2}{|c|}{$1^{\mathrm{a}}$ Fase $(\mathrm{s})$} & \multicolumn{2}{|c|}{$2^{\mathrm{a}}$ Fase $(\mathrm{s})$} \\
\hline & & Média \pm E.P. & Inibição (\%) & Média \pm E.P. & Inibição (\%) \\
\hline Controle (salina) & - & $85,12 \pm 2,75$ & - & $94,37 \pm 2,13$ & - \\
\hline $\mathrm{EH}$ & 100 & $46,25 \pm 1,47 * * *$ & 45,66 & $51,87 \pm 1,88 * * *$ & 45,03 \\
\hline $\mathrm{EH}$ & 200 & $30,87 \pm 1,48 * * *$ & 63,73 & $36,12 \pm 2,71 * * *$ & 61,72 \\
\hline EA & 100 & $45,37 \pm 2,15 * * *$ & 46,69 & $47,62 \pm 1,85 * * *$ & 49,54 \\
\hline EA & 200 & $34,12 \pm 1,66 * * *$ & 59,91 & $33,62 \pm 1,55^{* * *}$ & 64,37 \\
\hline $\mathrm{EE}$ & 100 & $34,00 \pm 2,10^{* * *}$ & 60,06 & $39,62 \pm 2,00 * * *$ & 58,02 \\
\hline $\mathrm{EE}$ & 200 & $32,50 \pm 1,68 * * *$ & 61,82 & $26,87 \pm 1,75^{* * *}$ & 71,53 \\
\hline Morfina & 1 & $18,12 \pm 1,49 * * *$ & 78,71 & $15,37 \pm 1,70 * * *$ & 83,71 \\
\hline
\end{tabular}

$* * * \mathrm{P}<0,001$. Significativos após análise de variância (ANOVA) seguido do teste de Student Newman-Keuls quando comparados ao controle.

Tabela 2. Efeitos dos extratos de Z. chiloperone sobre o edema de pata induzido por carragenina $(\mathrm{n}=6)$. Indometacina $(10 \mathrm{mg} / \mathrm{kg})$ foi o fármaco de referência.

\begin{tabular}{l|c|cc}
\hline Grupos experimentais & Doses $(\mathrm{mg} / \mathrm{kg})$ & \multicolumn{2}{|c}{ Volume do edema (mL) } \\
\cline { 2 - 4 } & & Média \pm E.P. & Inibição (\%) \\
\hline Controle (salina) & - & $1,00 \pm 0,07$ & - \\
EH & 100 & $0,57 \pm 0,05^{* * *}$ & 53 \\
EH & 200 & $0,43 \pm 0,05^{* * *}$ & 35 \\
EA & 100 & $0,65 \pm 0,04^{* *}$ & 48 \\
EA & 200 & $0,52 \pm 0,09^{* *}$ & 22 \\
EE & 100 & $0,78 \pm 0,05^{*}$ & 43 \\
EE & 200 & $0,57 \pm 0,06^{* * *}$ & 58 \\
Indometacina & 10 & $0,42 \pm 0,05^{* * *}$ &
\end{tabular}

$* \mathrm{P}<0,05, * * \mathrm{P}<0,01 \mathrm{e} * * * \mathrm{P}<0,001$. Significativos após análise de variância (ANOVA) seguido do teste de Student NewmanKeuls quando comparados ao controle. 
Tabela 3. Efeitos dos extratos de Z. chiloperone sobre o volume do exsudato da pleurisia induzida por carragenina $(\mathrm{n}=6)$. Indometacina $(10 \mathrm{mg} / \mathrm{kg})$ foi o controle positivo teste.

\begin{tabular}{l|c|cc}
\hline Grupos experimentais & Doses (mg/kg) & \multicolumn{2}{c}{ Volume do exsudato (mL) } \\
\cline { 2 - 4 } & & Média \pm E.P. & Inibição (\%) \\
\hline Controle (salina) & - & $2,43 \pm 0,14$ & - \\
EH & 100 & $1,58 \pm 0,09^{* * *}$ & 34,98 \\
EH & 200 & $1,27 \pm 0,08^{* * *}$ & 47,74 \\
EA & 100 & $1,55 \pm 0,09^{* * *}$ & 36,21 \\
EA & 200 & $1,37 \pm 0,12^{* * *}$ & 43,62 \\
EE & 100 & $1,63 \pm 0,08^{* * *}$ & 32,92 \\
EE & 200 & $1,40 \pm 0,08^{* * *}$ & 42,39 \\
Indometacina & 10 & $1,00 \pm 0,11^{* * *}$ & 58,85 \\
\hline
\end{tabular}

$* * * \mathrm{P}<0,001$. Significativos após análise de variância (ANOVA) seguido do teste de Student Newman-Keuls quando comparados ao controle.

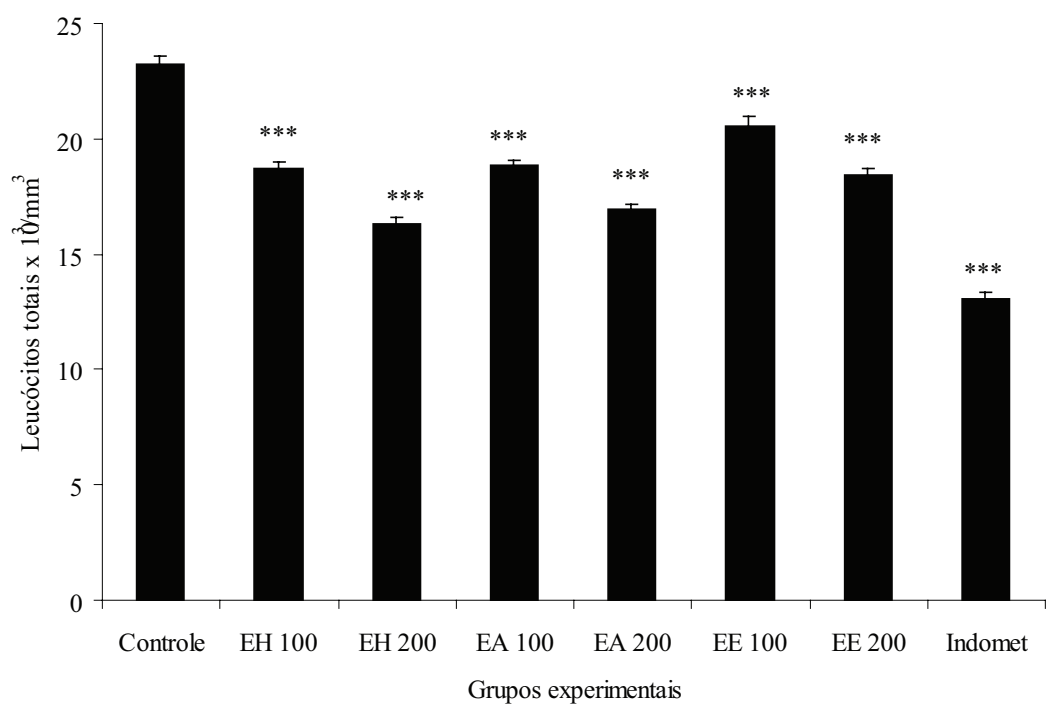

Figura 2. Efeitos dos extratos de Z. chiloperone sobre o $\mathrm{n}^{\mathrm{o}}$ de leucócitos totais do exsudato da pleurisia induzida por carragenina $(\mathrm{n}=6)$. Indometacina (Indomet), $10 \mathrm{mg} / \mathrm{kg}$, foi o controle positivo teste. ${ }^{* * *} \mathrm{P}<0,001$. Significativos após análise de variância (ANOVA) seguido do teste de Student Newman-Keuls quando comparados ao controle.

do tempo de lambida na $1^{\text {a }}$ fase (0-5 min) foi de 85,12 $\pm 2,75 \mathrm{~s}$ e na $2^{\mathrm{a}}$ fase (15-30 min) foi de $94,37 \pm 2,13 \mathrm{~s}$ para o grupo controle. Após 60 minutos de tratamento, as doses de 100 e $200 \mathrm{mg} / \mathrm{kg}$ inibiram ambas as fases. A dose de $200 \mathrm{mg} / \mathrm{kg}$ inibiu a primeira fase em 63,73\% (EH), 59,91\% (EA) e $61,82 \%$ (EE), enquanto a segunda fase foi inibida em $61,72 \%$ (EH), 64,37\% (EA) e 71,53\% (EE) $(p<0,001)$. A morfina $(1 \mathrm{mg} / \mathrm{kg})$ foi ativa em ambas as fases.

O efeito antiinflamatório dos extratos de $Z$. chiloperone avaliado pelo o método do edema de pata induzido por carragenina é mostrado na Tabela 2. O extrato EH inibiu, significativamente $(p<0,001)$, o edema de pata nas doses de $100 \mathrm{mg} / \mathrm{kg}(43,00 \%)$ e 200 $\mathrm{mg} / \mathrm{kg}(57,00 \%)$, enquanto o EA (p < 0,01) reduziu o edema em 35,00\% (100 mg/kg) e 48,00\% (200 mg/kg). Este efeito também foi demonstrado por EE que causou $22,00 \%(100 \mathrm{mg} / \mathrm{kg})(\mathrm{p}<0,05)$ e $43,00 \%(200 \mathrm{mg} / \mathrm{kg})(\mathrm{p}$ $<0,001)$ de inibição sobre o edema de pata em relação ao controle. A indometacina causou 58,00\% $(0,42 \pm 0,05)$ de redução do edema de pata.

Os efeitos sobre a pleurisia demonstraram que as doses dos extratos reduziram, significativamente $(\mathrm{p}<$ 0,001 ), o volume do exsudato e o $\mathrm{n}^{0}$ de leucócitos totais (Tabela 3 e Figura 2). A dose de $200 \mathrm{mg} / \mathrm{kg}$ diminuiu o volume do exsudato em $47,74 \%$, 43,62\% e 42,39\% para EH, EA e EE, respectivamente. A inibição da migração leucocitária também foi significativa $(\mathrm{p}<0,001)$ na dose de $200 \mathrm{mg} / \mathrm{kg}$ com 29,83\% (EH), 26,81 (EA) e 20,69\% (EE). Não houve diferença significativa entre polimorfonucleares e mononucleares em relação ao controle. A indometacina reduziu o volume do exsudato e a migração leucocitária.

\section{DISCUSSÃO}

Os extratos das folhas de Z. chiloperone possuem atividades antinociceptivas sobre o sistema 
nervoso central e periférico que foram demonstradas pelos efeitos inibitórios das contorções abdominais e dos tempos de lambida da pata (Figura 1 e Tabela 2). A segunda fase do teste da lambida da pata é também indicativa de uma possível atividade antiinflamatória.

Os mecanismos envolvidos na indução da dor estão relacionados a substâncias bioativas liberadas pelo sistema nervoso simpático, a metabólitos da via do ácido araquidônico (Duarte et al., 1992) e ao sistema opióide (Collier et al., 1968). Ao reduzir as contorções abdominais, os extratos testados, possivelmente, estão exercendo ações periférica e central pela inibição dos mediadores endógenos (Collier et al., 1968). A primeira fase do tempo da lambida é caracterizada pela propagação imediata de impulsos para o sistema nervoso central. Ao agir nos receptores opióides a morfina reduz o tempo nesta fase (Shibata et al., 1989). Após 15 minutos de aplicação, a formalina induz um processo inflamatório local que pode ser inibido por antiinflamatórios tais como indometacina. A redução do tempo de lambida nesta fase pode ser devido à diminuição da biossíntese de prostaglandinas (Farsam et al., 2000). Os resultados do teste da lambida confirmam os obtidos nas contorções abdominais.

A atividade antiinflamatória indicada pelo teste da formalina foi confirmada, avaliando os efeitos antiedematogênicos dos extratos de Z. chiloperone no modelo do edema de pata induzido por carragenina (Tabela 2). A formação do edema é ocasionada pela produção de prostaglandinas após aplicação da carragenina que é um agente inflamatório (Farsam et al., 2000). Houve uma redução bastante significativa do edema, demonstrando que, provavelmente, os constituintes dos extratos estão inibindo o processo inflamatório.

A injeção intrapleural de carragenina ocasiona a formação do exsudato na cavidade pleural (Ammendola et al., 1975; Almeida et al., 1980) e a migração leucocitária (Compasso et al., 1975; Almeida et al., 1980). É um método bastante interessante, pois avalia o infiltrado inflamatório e confirma os resultados obtidos no edema de pata. Os antiinflamatórios nãoesteróides, tal como a indometacina, inibem o acúmulo do exsudato e mobilização de leucócitos entre 3 e 6 horas após carragenina (Vinegar et al., 1973; Almeida et al., 1980). Ao reduzir o volume do exsudato e a migração leucocitária, os extratos de Z. chiloperone corroboraram com os resultados do edema de pata (Tabela 3 e Figura 2).

Os extratos das folhas de Z . chiloperone possuem constituintes ativos com atividades antinociceptiva e antiinflamatória como demonstrado nos métodos empregados, sugerindo suas potencialidades para fins terapêuticos. Os resultados confirmam as informações sobre os usos populares de Z. chiloperone.

\section{AGRADECIMENTOS}

Nossos agradecimentos ao CNPq a UFJF pelas bolsas de iniciação científica.

\section{REFERÊNCIAS}

Albuquerque GB, Rodrigues RR 2000. A vegetação do Morro de Araçoaiaba, Floresta Nacional de Ipanema, Iperó (SP). Scientia Florestalis 58: 145-159.

Almeida AP, Bayer BM, Horakova Z, Beaven MA 1980. Influence of indomethacin and other anti-inflammatory drugs on mobilization and production of neutrophils: studies with carrageenan-induced inflammation in rats. J Pharmacol Exp Ther 214: 74-79.

Amaral FMM, Ribeiro MNS, Barbosa-Filho JM, Reis AS, Nascimento FRF, Macedo RO 2006. Plants and chemical constituents with giardicidal activity. Rev Bras Farmacogn 16(Supl.): 696-720.

Ammendola G, Di Rosa GM, Sorrentino L 1975. Leucocyte migration and lysosomal enzymes release in rat carrageenin pleurisy. Agents Actions 5: 250-255.

Arruda MSP, Fernandes JB, Vieira PC, Silva MFGF, Pirani JR 1992. Chemistry of Zanthoxylum rhoifolium: A new secofuroquinoline alkaloid. Biochem Syst Ecol 20: 173-178.

Chung Y-C, Chien C-T, Teng K-Y, Chou S-T 2006. Antioxidative and mutagenic properties of Zanthoxylum ailanthoides Sieb \& Zucc. Food Chem 97: 418-425.

Collier HO, Dinneen LC, Johnson CA, Schneider C 1968. The abdominal constriction response and its suppression by analgesic drugs in the mouse. Brit $J$ Pharmacol Chemother 32: 295-310.

Compasso F, Dunn CJ, Yamamoto S, Willougbby DA, Giroud JP 1975. Further studies on carrageenan-induced pleurisy in rats. $J$ Pathol 116: 117-124.

Diehl EE, von Poser GL, Henriques A T 2000. Constituents of Zanthoxylum rugosum St.-Hil \& Tul. Biochem Syst Ecol 28: 275-277.

Duarte IDG, Ferreira-Alves DL, Nakamura-Craig M 1992. Possible participation of endogenous opioid peptides on the mechanism involved in analgesia by vouacapan. Life Sci 50: 891-897.

Facundo VA, Silveira ASP, Braz-Filho R, Pinto AC, Rezende CM 2005. Constituintes químicos de Zanthoxylum ekmanii (URB.) Alain. Quim Nova 28: 224-225.

Farsam H, Amanlou M, Dehpour AR, Jahaniani F 2000. Antiinflammatory and analgesic activity of Biebersteinia multifida DC. root extract. J Ethnopharmacol 71: 443447.

Ferreira ME, Rojas de Arias A, Ortiz ST, Inchausti A, Nakayama H, Thouvel C, Hocquemiller R, Fournet A 2002. Leishmanicidal activity of two canthin-6one alkaloids, two major constituents of Zanthoxylum chiloperone var. angustifolium. J Ethnopharmacol 80: 199-202.

Ferreira ME, Nakayama H, de Arias AR, Schinini A, de Bilbao NV, Serna E, Lagoutte D, Soriano-agaton F, Poupon E, Hocquemiller R, Fournet A 2007. Effects of canthin- 
6-one alkaloids from Zanthoxylum chiloperone on Trypanosoma cruzi-infected mice. J Ethnopharmacol 109: 258-263.

Gessler MC, Nkunya MHH, Mwasumbi LB, Heinrich M, Tanner M 1994. Screening Tanzanian medicinal plants for antimalarial activity. Acta Tropica 56: 65-77.

Guy I, Charles B, Guinaudeau H, Ferreira ME, Arias AR, Fournet A 2001. Essential oils from Zanthoxylum chiloperone and $Z$. riedelianum growing in Paraguay. Pharm Biol 39: 152-154.

Hunskaar S, Hole K 1987. The formalin test in mice: dissociation between inflammatory and non-inflammatory pain. Pain 30: 103-114.

Jullian V, Bourdy G, Georges S, Maurel S, Sauvain M 2006. Validation of use a tradicional antimalarial remedy from French Guiana, Zanthoxylum rhoifolium Lam. J Ethnopharmacol 106: 348-352.

Koster R, Anderson M, Beer EJ 1959. Acetic acid for analgesic screening. Federation Proceedings 18: 412.

Milliken W 1997. Plants for malaria for fever, medicinal species in Latin America. London: The Royal Botanical Garden.

Oliveira EL, Freitas PC, Guedes MLS, Velozo ES 2002. Estudo fitoquímico de Zanthoxylum stelligerum (Turcz). Rev Bras Farmacogn 12(Supl.): 29-30.

Rahman MT, Alimuzzaman M, Ahmad S, Chowdhury AA 2002. Antinociceptive and antidiarrhoeal activity of Zanthoxylum rhetsa. Fitoterapia 73: 340-342.

Shibata M, Ohkubo T, Takahashi H, Inoki R 1989. Modified formalin test: characteristic biphasic pain response. Pain 38: 347-352.

Silva LA, Soares JJ 2003. Composição florística de um fragmento de floresta estacional semidecídua no município de São Carlos - SP. Rev Árvore 27: 647-656.

Soriano-Agatón F, Lagoutte D, Poupon E, Roblot F, Fournet A, Gantier J-C, Hocquemiller R 2005. Extraction, hemisynthesis and synthesis of canthin-6-one analogues. Evaluation of their antifungical activities. J Nat Prod 11: 1581-1587.

Stermitz FR, Sharifi IA 1977. Alkaloids of Zanthoxylum monophyllum and Z. punctatum. Phytochemistry 16: 2003-2006.

Talapatra SK, Dutta SK, Talapatra B 1973. Alkaloids and terpenoids of Zanthoxylum ovalifolium. Phytochemistry 12: 729-730.

Tane P, Wabo HK, Connolly JD 2005. A new benzophennanthridine alkaloid from Zanthoxylum buesgenii. Fitoterapia 76: 656-660.

Tatsadjieu LN, Essia Ngang JJ, Ngassoum MB, Etoa F-X 2003. Antibacterial and antifungal activity of Xylopia aethiopica, Monodora myristica, Zanthoxylum xanthoxyloïdes and Zanthoxylum leprieurii from Cameroon. Fitoterapia 74: 469-472.

Thouvenel C, Gantier JC, Duret P, Fourneau C, Hocquemiller R, Ferreira ME, Rojas de Arias A, Fournet A 2003. Antifungal compounds from Zanthoxylum chiloperone var. angustifolium. Phytother Res 17: 678-680.
Vigneron M, Deparis X, Deharo E, Bourdy G 2005. Antimalarial remedies in French Guiana: a knowledge attitudes and pratices study. J Ethnopharmacol 98: 351-360.

Vinegar R, Traux JF, Selph JL 1973. Some quantitative temporal characteristic of carrageenin-induced pleurisy in the rat. Proc Soc Exp Bio Med 143: 711-714.

Weenen H, Nkunya MHH, Bray DH, Mwasumbi LB, Kinabo LS, Kilimali VAEB, Wilnberg JBPA 1990. Antimalarial compounds containing an alpha,beta-unsaturated carbonyl moiety from Tanzanian medicinal plants. Planta Med 56: 371-373.

Winter CA, Risley EA, Nuss GW 1962. Carrageenin-induced edema in hind paw of the rat as an assay for antiinflammatory drugs. Proc Soc Exp Biol Med 111: 544-547. 